\title{
Quels objets numériques pour accompagner la conception dans des projets d'innovation?
}

Céline Verchère et Laurent Collet

\section{(2) OpenEdition}

\section{Journals}

Édition électronique

URL : http://journals.openedition.org/anthrovision/647

DOI : 10.4000/anthrovision.647

ISSN : 2198-6754

Éditeur

VANEASA - Visual Anthropology Network of European Association of Social Anthropologists

Référence électronique

Céline Verchère et Laurent Collet, « Quels objets numériques pour accompagner la conception dans des projets d'innovation? », Anthrovision [En ligne], 2.1 | 2014, mis en ligne le 15 septembre 2014, consulté le 21 avril 2019. URL : http://journals.openedition.org/anthrovision/647 ; DOI : 10.4000/ anthrovision. 647

Ce document a été généré automatiquement le 21 avril 2019.

(c) Anthrovision 


\title{
Quels objets numériques pour accompagner la conception dans des projets d'innovation?
}

\author{
Céline Verchère et Laurent Collet
}

\section{Introduction}

En 2009, l'Union Européenne entrait dans son « année de la créativité et de l'innovation ». Comme beaucoup d'Etats ou d'entreprises auparavant, elle proclamait ainsi haut et fort son engagement dans "l'économie de l'innovation" et dans la "démocratie technique« (Von Hippel 2005). Depuis une quinzaine d'années en effet, l'innovation est appréhendée comme le moteur indispensable des entreprises, ce qui a eu pour conséquence d'étendre les domaines habituellement dédiés à ce type de structure : ainsi la R\&D technologique, bien qu'incontournable, n'a plus été considérée comme le seul élément permettant de favoriser l'avènement d'innovations. Sont venus s'ajouter toute une série de métiers et de compétences (marketing, design, ergonomie, ethnométhodologie) qui tendent essentiellement vers un but : réduire "l'incertitude« et "anticiper les risques d'échec" liés au développement des innovations, à toutes les étapes de la conception (de l'idée jusqu'au marché). C'est en ce sens que les sciences humaines et sociales (SHS) ont été appelées à intervenir dans les activités de R\&D. Parmi elles, la sociologie, ou plus spécifiquement les "approches usages" sont venues compléter les démarches plus traditionnelles, dans le but de favoriser la conception d'innovations technologiques. Ce tournant s'est avéré d'autant plus nécessaire pour les entreprises qu'elles ont dû apprendre à composer avec une nouvelle donnée : »le grand public« qui s'exprime de plus en plus en amont dans les étapes de réalisation des produits (plate-forme collaborative, tchats, forums...). C'est en partie ce qui explique que les nouvelles pratiques de conception accordent une place croissante aux porte-paroles des usagers (associations, consultants), autant qu'aux "utilisateurs potentiels" des innovations à venir. Ces approches par projet, dites "agiles" et itératives, considèrent que les utilisateurs potentiels doivent être partie prenante de la dynamique de conception et amènent, à leur 
manière, une expertise qui sert le projet et, dans une certaine mesure, "co-construit la connaissance«. Pour certains sociologues, l'entrée dans ce monde a marqué "un tournant praticien« (Piriou 2008) qui les a amenés à développer de nouvelles pratiques, au plus près des laboratoires technologiques, en amont des processus de conception et loin du produit "final«. La question se pose d'emblée : avec les outils conceptuels et méthodologiques que le sociologue tient à sa disposition, comment peut-il traiter et analyser un phénomène qui est "potentiellement susceptible d'advenir« (en ce sens où, au moment où l'innovation émerge dans les laboratoires, ni les utilisateurs, ni les marchés n'existent) ? Pour reprendre une image simple : avec quels outils aurait-il pu observer et accompagner l'avènement de l'I Phone (et prévoir son succès) avant même que celui-ci n'ait pris forme, voire avant même que l'idée n'ait germé, au moment où il n'avait tout au plus qu'un capteur devant les yeux ? Voilà tout le dilemme auquel ces sociologues sont confrontés. La difficulté supplémentaire tient à »'obligation « d'agir en interdisciplinarité, dans la mesure où le processus de conception est peuplé d'ingénieurs qui n'ont qu'un objectif en tête : parvenir à un démonstrateur (démontrer que des fonctions marchent, indépendamment de la forme de l'objet à venir), à une maquette (dispositif non fonctionnel, qui donne à voir), voire à un prototype (dispositif fonctionnel permettant au minimum de tester l'objet ou le service final afin de l'améliorer et d'entrer en phase de production industrielle). C'est dans ce contexte de nécessité d'obtenir $d u$ concret que la conception se fait « autour d'un instrument, d'un objet ou d'un terrain » et que " la mobilisation de ressources de plusieurs disciplines s'impose parfois. " (Vinck, 2007 : 84). Le sociologue, s'il veut influer d'une manière ou d'une autre sur le processus, doit dialoguer avec ces compétences, sachant qu'il n'est pas indispensable à la réalisation de l'objectif. Dernière difficulté enfin, comment, donc, infléchir le processus et plus largement, comment traiter la matière analysée pour qu'elle puisse être appropriée par des individus qui n'appartiennent pas à la communauté des "pairs" et qui ne raisonnent pas d'après les mêmes paradigmes ? Tout l'enjeu consiste à faire vivre le raisonnement sociologique qui se fait par problématisation (contextualisation d'une question théorique), là où les raisonnements technologiques fonctionnent le plus souvent par modélisation (c'est-à-dire par la recherche d'un cas typique dénué de toute contextualisation). C'est dans ce contexte que l'équipe de UMAN LAB $^{1}$ a travaillé pendant trois ans au sein du MINATEC CEA Grenoble ${ }^{2}$. Les sociologues praticiens sont donc partis dans l'expérimentation des méthodes et outils, d'abord pour aborder de front la pratique du travail "en interdisciplinarité«, puis pour défricher ces territoires "nouveaux", en ne travaillant plus sur des usages réels mais sur des usages à venir (nous les appelons les "usages projetés «). Peu à peu, ils se sont mis à élaborer et construire des représentations d'usages fictifs (projection d'usages) qu'ils ont mis en scène, avec des usagers en prise avec les innovations technologiques dans des contextes variés (transports, santé, gestion de l'énergie, habitat...). Ces différentes représentations ont été d'abord décrites et rendues sous forme de rapports. Elles ont été aussi mises en récit (sous forme de scénarios d'usage), ou encore racontées sous forme de films. De fait, le présent article vise d'abord à présenter quelques-uns de ces »documents formels« en les resituant dans des projets de R\&D, en précisant la posture méthodologique et épistémologique que soustendent ces pratiques. Une analyse réflexive montrera à quelles limites ont été confrontés ces praticiens: cadrer le sens de leurs représentations afin de permettre l'intercompréhension des partenaires, y compris celui des utilisateurs finaux, dans le contexte d'une action orientée vers le succès (Habermas 1978). Or, dans la réalité des projets, cette double contrainte (intercompréhension et succès) s'avère être paradoxale : 
le succès de l'intercompréhension passe par une confrontation des complexités, ce qui peut nuire au succès du projet lui-même ; et dans le même temps, la recherche du succès peut passer outre la nécessité d'intercompréhension entre acteurs. Enfin, nous terminerons par plaider la cause du travail en interdisciplinarité, comme "garde-fou méthodologique" dans des projets où la "convergence" des pratiques s'imposent d'emblée, tant sur la démarche que sur les documents produits et les objectifs visés.

\section{Les usages projetés : formes et fondements}

\section{Des méthodologies pour accompagner la conception?}

2 Les processus de conception se concentrent généralement sur trois grandes étapes, visant chacune à faire progresser l'idée, pour qu'elle passe du statut de germe, à celui d'invention puis d'innovation : une première phase s'attache à l'exploration et à la découverte, puis une phase se concentre sur la conception, et enfin vient le temps du développement. Ces phases ne sont pas linéaires, elles peuvent être "activées" à n'importe quel moment du cycle de conception, selon la maturation du processus. C'est dans ces grands moments que s'inscrit l'approche dite "usage" (et plus largement "user centric «). S'appuyant sur des méthodologies issues des sciences humaines et sociales (de type ethnologie, observation participante, enquêtes qualitatives et quantitatives), cette démarche explore toute une série de méthodes visant à servir la conception, autrement dit à "optimiser" le processus de conception. A chaque phase correspondent des méthodes, mais aussi des types de »rendus«.

Du côté de l'équipe des "sociologues praticiens", plusieurs écueils sont vite venus questionner les pratiques initiales, qui consistaient à mener les »enquêtes« ou faire la "veille« au cours du projet, puis à rendre un »livrable« sous forme d'un document écrit. En effet, nous avons constaté à plusieurs reprises que ces documents n'étaient pas lus, ou s'ils l'étaient, que les destinataires ne s'avaient qu'en faire : en bref, ils n'avaient pas d'effet sur le processus de conception. L'équipe a alors décidé de modifier ses modes de rendus, en profitant de l'opportunité que pouvait apporter les supports multimédia ou tout simplement certains supports "tangibles". De notre point de vue, la nature de ces supports pouvait permettre de communiquer le travail à des publics divers, experts et technologues et d'avoir plus d'impact sur le processus d'innovation, par sa force d'évocation du réel à venir. C'est ainsi que les sociologues se sont mis à rendre, sur le mode »expérientiel«, des documents aux formats inhabituels (par exemple un support DVD ou encore un jeu de carte comme livrables de projet). Cependant, le travail sur les "projections d'usages" a conduit à l'édification d' "un cimetière assumé de démos en tout genre » où se peuplent des "représentations intermédiaires de concepts - scénario, démo, maquette [...] « qui »sont quelques-unes des modalités de ce nouveau paradigme de la conception" (Trompette and Blanco, 2009 : 105). Ainsi, ont été réalisés des "concepts«, des »scénarios d'usage«, des "supports multimédias«, en amont de la phase de conception visant à la preuve de concept. Divers documents "formels" circulaient donc, au sein de deux réseaux: l'équipe de conception (ingénieurs, ergonomes, sociologues, chercheurs, etc.), mais aussi une sphère élargie, c'est-à-dire des d'utilisateurs dits "potentiels" qui étaient appelés à réagir sur ce qui leur étaient montré. 
Figure 1 : Exemple de support utilisé pour la conception

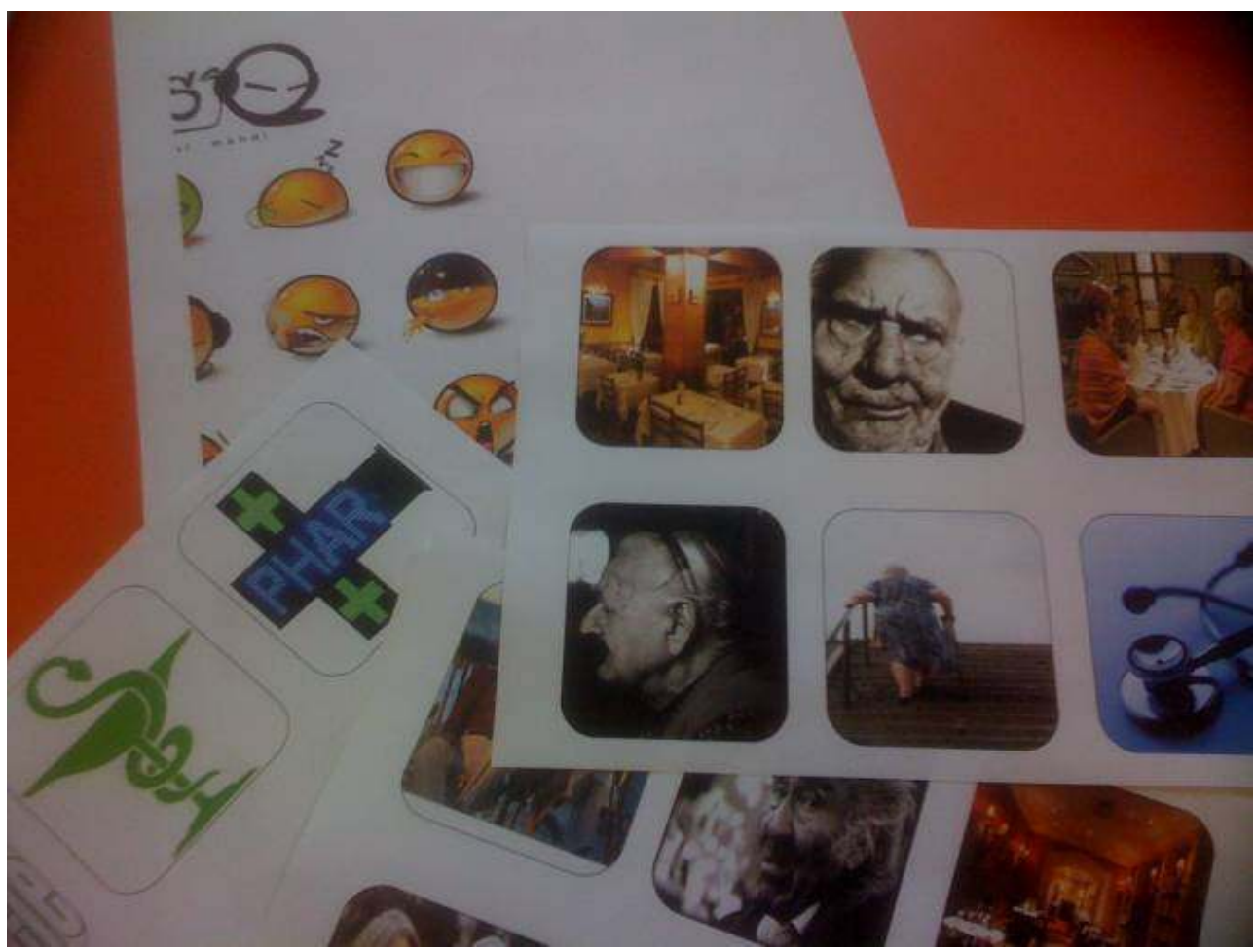

Source : CEA Grenoble

4 Chacun de ces supports, dans une certaine mesure, visent à "stabiliser l'incertain «, fixer des représentations à un moment donné, un peu à la manière de l'architecte qui dessine les plans d'une maison afin de lui donner enfin corps à partir de la vie que ses habitants veulent y mener (appartement ou maison sur-mesure) ou doivent y mener (versions standards). Ces matériaux cherchent à "nourrir« le travail de conception, notamment en permettant l'expression de points de vue internes ou externes aux projets.

5 Nous pouvons résumer nos types d'intervention et de rendus aux schémas suivants : 
Figure 2 : Types d'intervention et rendus associés

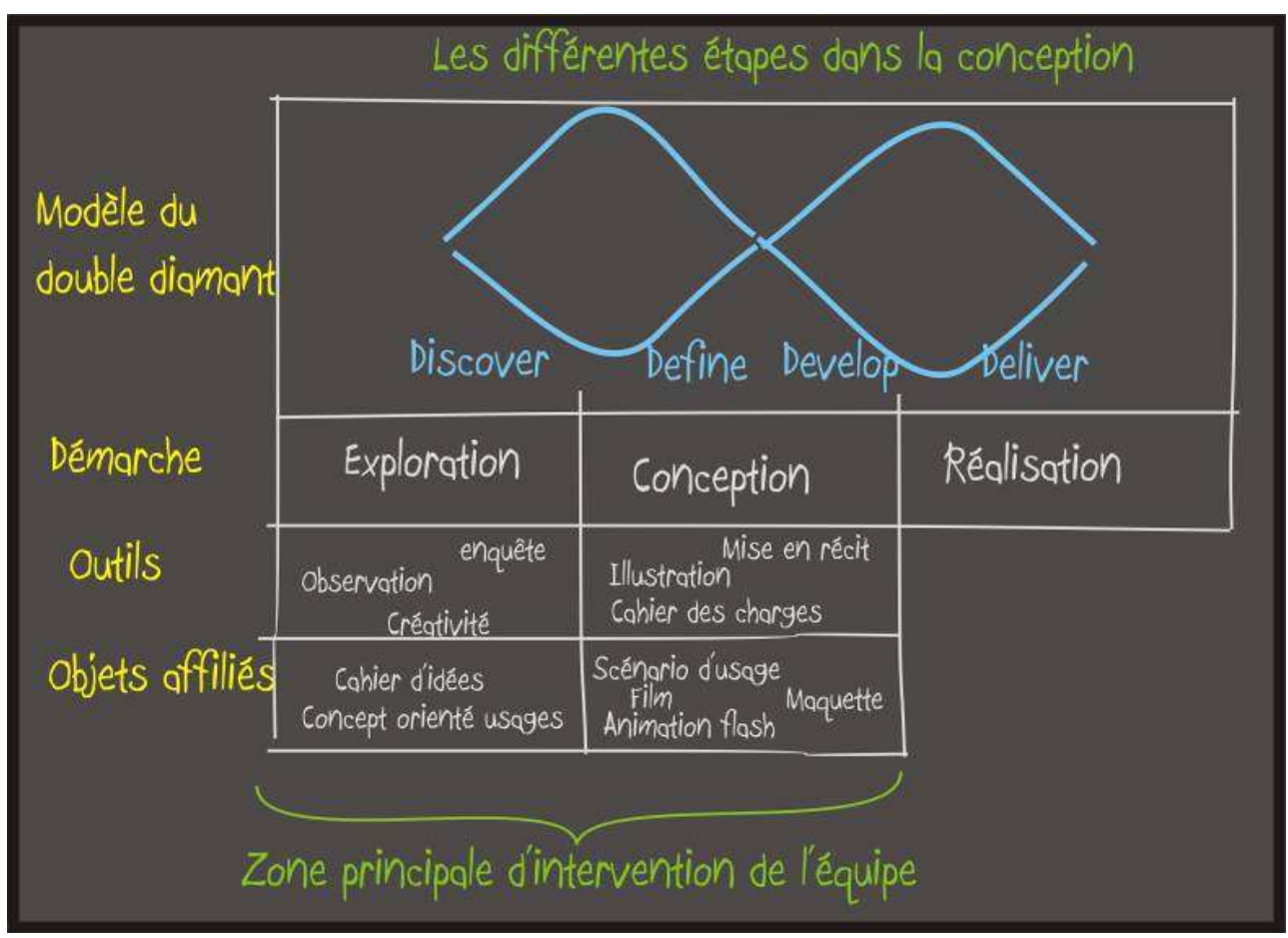

Source : CEA Grenoble

\section{Spécificité de l'approche et posture des praticiens}

\section{Approche spéculative et posture internaliste}

6 A l'origine, il existe peu d'ouvrages qui traitent directement de la question des usages appliquée à la conception d'innovation technologique. Il s'agit plutôt d'un »collège invisible« (Joüet 2000 ; Le Marec 2001) qui s'est constituée depuis les années 70 en France, dans la discipline sociologique essentiellement, autour de la question large des usages.

Bien qu'il existe désormais une littérature plus abondante venant éclairer cette question, la démarche est avant tout "expérientielle« : nous pourrions dire qu'elle est de nature spéculative : elle vise à formuler des »choix de conception«. Les méthodes servent le même dessein : le résultat de leur mise en œuvre doit permettre d'orienter la conception. En ce sens, dans le vieux débat entre posture externaliste et posture internaliste, l'approche passe plutôt du côté des internalistes : la démarche ne vise pas tant la recherche de la vérité (par la recherche d'effets que nous pourrions relier à des causes possibles et par là même rendre généralisables), que la recherche d'opérationnalité, où, par une entreprise de co-construction de la connaissance, les sciences humaines "guident" les choix de conception, au même titre que d'autres sciences ou approches technologiques (road maps). D'après Patenaude, J. et Boissy, P., il s'agit d'une posture justificative plutôt qu'explicative : »en effet, la technoscience pose que, sur le plan symbolique, la science intègre dans son projet scientifique non seulement sa dépendance envers des techniques hypercomplexes comme instruments de la méthode scientifique, mais que sa finalité est elle-même opératoire (OGM, nanotechnologies, etc.)« (Patenaude et Boissy, 2009 : 85). D'où l'importance de l'accompagnement comme visée, où la finalité 
des sciences humaines est elle-même opératoire. D'après ces auteurs encore, »il ne s'agit pas de décrire ou d'expliquer le réel, mais plutôt d'assister le processus de transformation en identifiant et en évaluant les enjeux de l'intérieur du processus lui-même« (2009:85). Il s'agit en quelque sorte d'une philosophie du savoir dans l'action, qui demande à chaque acteur d'être dans une posture de réflexivité par rapport à son champ de recherche, afin de pouvoir s'ouvrir à d'autres domaines et d'accepter que des "perturbations" viennent modifier la trajectoire des processus tels que pensés initialement. Par conséquence, les méthodes mobilisées s'inscrivent dans cette posture : elles visent à provoquer la discussion autour du "vraisemblable« plutôt que de "la vérité«, ou dit autrement, à rendre légitime et cohérent un imaginaire partagé.

\section{Un travail de création}

Une autre singularité de l'approche est que le sociologue est amené, littéralement, à "créer" la matière qu'il va ensuite analyser. En quelque sorte, il est amené à "représenter les représentations« . En effet, puisqu'aucun phénomène n'est observable, puisque ce phénomène n'est pas encore arrivé (et arrivera-t-il seulement ?), il se doit de "'imaginer«, ce qu'il fait avec divers types de médiations : les scénarios d'usage, etc. Il est ainsi amené à donner corps à des personnages, des scènes, des dispositifs qu'il testera ensuite afin d'en recueillir un matériau possible pour l'analyse. Sa démarche vise non seulement à créer des histoires mais également à les rendre crédibles par des descriptions d'objets, de personnes, de lieux, afin de faire croire que "tout ceci« existe car "tout ceci« est vraisemblable et cohérent. Il produit, peut-être sans le savoir, des effets de réel (Barthes 1968). Le sociologue devient donc auteur de fiction (sans tomber dans la sciencefiction, mais se tenant à sa porte), ce qui n'est pas sans poser question, notamment sur les limites auquel il s'astreint entre objectivation et subjectivité, analyse à »froid« et propositions imaginaires associées.

\section{La manipulation de supports inédits : le multimédia}

9 Troisième "nouveauté« dans sa pratique, le sociologue opère avec des outils multimédia, c'est-à-dire qu'il manipule non seulement du texte, mais aussi des images, du son et des »hyper-liens«. Là encore, il va devoir »créer« une histoire, donner corps en »2D« à ce qu'il a l'habitude de retranscrire en 1D (la feuille). Les formes de médiatisations possibles sur document numérique des pratiques et des imaginaires sociaux sont multiples : images fixes ou animées (flash), textes, animations multimédias, etc. Il est à noter que dans les projets sur lesquels il travaille, il n'existe pas encore de forme figée, standardisée, reconnue et reconnaissable par les différents acteurs d'un projet. Dès lors, l'innovation se situe également dans la démarche de recherche qui prend des formes d'expression diverses. Le sociologue fait souvent appel à ses connaissances "privées » de produits médiatiques : films linéaires ou interactifs, animations flash, romans, etc. Il pratique alors une forme d'importation sauvage de connaissances dans son monde professionnel. De fait, la posture visant à accompagner les processus de conception, au même titre que l'entrée dans un travail sur les "usages projetés « modifie profondément la démarche de ces sociologues "praticiens". "Les sociologues s'écartent du cœur de leur territoire, la production immatérielle et écrite, pour expérimenter de nouveaux terrains et de nouvelles formes d'expression de leur connaissance, passant notamment par un travail « animé » sur l'imaginaire. Ils promettent aussi l'opérationnalité des outils proposés dans 
la démarche« (Verchere and Anjembe 2010). Ce faisant, ils relèvent un triple défi : opérer un travail sur un matériau nouveau (l'imaginaire), composer avec des supports inédits (les supports multimédia) et enfin privilégier une démarche qui s'éloigne des "règles de la méthode« habituellement appliquées dans leur domaine. Nous allons voir que cela n'est pas sans poser problème.

\section{Retours réflexifs par « l'analyse des »traces« »}

La création de différents "médiatisations multimédias (textuelles, sonores et visuelles) « porte l'espoir d'une meilleure intercompréhension des pratiques et des imaginaires sociaux de tous les acteurs, afin d'arriver à créer les conditions d'un espace de discussion gouverné par la Raison et visant le bien commun (Habermas 1978), et d'éviter les effets d'imposition par un seul acteur. Or, force fut de constater que plusieurs problèmes virent le jour, sans que la méthodologie mobilisée, les supports ou encore le dialogue puissent modifier la donne. Dans les pires des cas, les supports ont pu même ajouter du risque aux risques déjà induits par le projet.

\section{L'analyse par les »objets intermédiaires"}

\section{Des documents multimedia qui peuvent parasiter le travail en commun}

11 D'abord, au sein même des projets et entre les différents collaborateurs, ces »objets intermédiaires« (scénario multimedia, petits films, etc.), loin de susciter l'adhésion et la compréhension, semblaient pour certains au contraire éloigner les »communautés de pratique« agissantes. Sans entrer dans le détail de l'analyse, la mobilisation du concept d'objet frontière (Bowker and Star 1999) et plus précisément de son cousin le concept de "Boundary Negotiating Artifact» (Lee 2007) nous a permis de comprendre de manière dynamique les difficultés suscitées par l'élaboration de tels dispositifs. Typiquement, sur ce projet étudié, les scénarios d'usage, rédigé par l'équipe des sociologues, n'ont pas été discutés par les technologues lorsqu'ils ont été élaborés (Verchère et Anjembe, 2010). De fait, les supports multimédia n'ont pas pu être appréhendés par cette équipe qui, peu à peu, a désinvesti le projet et s'est mise en retrait. Comme l'a dit un des membres du projet: "ce qu'on voit qui s'est passé là, c'est que les SHS étaient devenues à la fois producteurs et récepteurs d'elles-mêmes" (enquête réflexive sur le projet, UMAN LAB, 2009). Ainsi, les objets créés peuvent être sources d'exclusion, ne servant au final que leurs créateurs et leurs causes. L'inverse fut aussi vrai pour les sociologues, qui ont désinvesti l'élaboration de la maquette, considéré comme le terrain de jeu des technologues par excellence. Ainsi, les objets réalisés au cours du projet n'ont pas réussi à "brouiller les cartes" professionnelles et proposer de nouvelles manières de travailler ensemble. Il y a de fait tout un travail de déconstruction à mener afin d'éclairer le rôle de ses objets au sein des projets : qu'ils soient objets visant l'intercompréhension entre experts, objets visant l'intercompréhension des pratiques sociales, objets visant à persuader, etc.

\section{L'illusion d'un langage commun}

De plus, le dialogue, et plus largement la question du »langage commun« autour d'un projet apparut vite comme dépourvu de réalité. Bien au contraire, pour chaque projet, il semble se créer un langage "créole« et »situé« (Anjembe 2012), où les terminologies ne 
sont comprises et échangées que par ses membres et demeurent non reproductibles pour des projets ultérieurs. Par ailleurs, le travail en transdisciplinarité finit par »délocaliser « les concepts de leur ancrage disciplinaire et ils sont utilisés par les membres du projet uniquement comme un référent pour le projet, connus d'eux seuls. Ce savoir acquis ne dépasse donc pas l'espace du projet. Les films ou les supports multimédias ne semblent pas modifier cet état de fait.

Extrait de projet $X$ - conférence téléphonique

Membre projet : »mais use case et scénario d'usage, c'est synonyme, non ? (rires) «

Umanlab : »oui, enfin, c'est pas la même langue... «

Chef de projet: "ça c'est sûr, mais bon, faut quand même qu'on parle la même

langue pour savoir si on parle de choses différentes (rires).«

\section{D'étranges réactions dans les focus group (réunions de groupe).}

Enfin, quelques "étrangetés« sont venues là encore nous poser question. L'équipe de sociologues a pris l'habitude de réaliser des focus group (réunions de groupe) afin de montrer des petits films à des «utilisateurs potentiels». Sur ce projet (www.ist-esense.org/En cache - Pages similaires), plusieurs films déclinaient la même thématique (sécurité et capteurs), prise sous un angle différent. Or, nous avons constaté qu'un des films suscitait plus de réactions hostiles que les autres, alors que le canevas du scénario était construit à l'identique. La traditionnelle «analyse de corpus » ne permettait pas d'éclairer cette différence de réactions constatées au sein du focus group.

\section{L'approche par la question du sens des images}

14 Le scénario a été initialement rédigé par l'équipe de »sociologues praticiens« du projet, puis la réalisation et le montage du film ont été sous-traités. Dans le cas de ce projet, si on y regarde de plus près, le sens des images a échappé aux présumés "scénaristes et réalisateurs", à savoir les sociologues. Le sociologue s'est retrouvé à devoir construire des "récits de vie« sans une réelle maîtrise de l'art de construire les récits et à produire des documents multimédias sans connaissance des spécificités sémiotiques des écritures.

Figure 3 : Le film relatant une des histoires du projet "E sense»

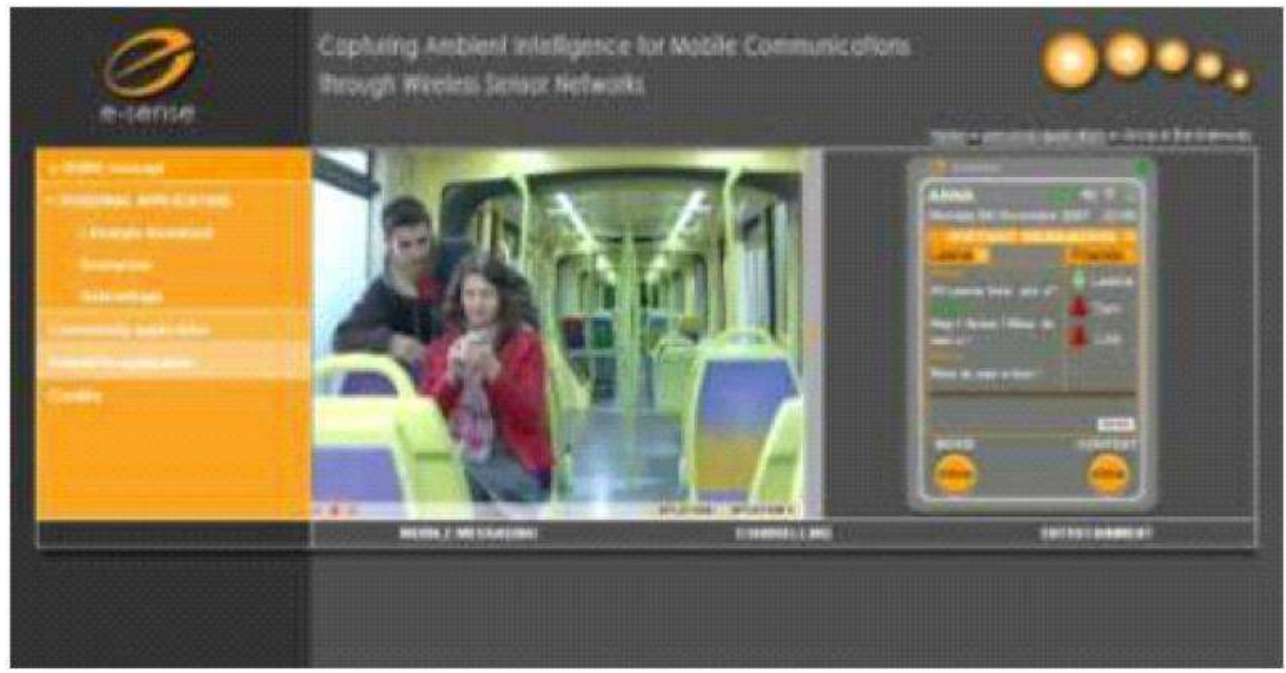

Source= http://mac-serv.univ-tIn.fr/rema/partage/anna_in_the_tramway.swf 

individuel d'alerte en cas d'agression dans les transports en commun. Une histoire ${ }^{3}$ a été ainsi élaborée sous forme de récit interactif proposant deux parcours mettant en scène les personnages d'une femme d'une quarantaine d'année et d'un jeune homme d'une vingtaine d'année :

- un parcours où une femme se fait arracher des mains son téléphone, déclenchant une alerte dans le tramway, faisant fuir l'agresseur,

- un parcours où elle utilise la fonction visiophone de son mobile pour communiquer avec son fils, qui, dans l'histoire, se trouve être un copain du second personnage, et qui en profite pour draguer. Dans cette scène, l'alerte ne se déclenche pas.

Cette structuration pose un problème si l'on prend connaissance des deux parcours dans n'importe quel ordre. Voilà ce qui est donné à voir : dans les transports en commun, une femme se fait 1) soit agresser, 2) soit draguer. Il y a donc un premier niveau de problème, que génère l'interactivité. Avec le »chemin de fer« proposé, non linéaire, le sens de lecture de l'histoire n'est pas maitrisé. Le second problème se situe au niveau visuel. L'analyse des images véhiculées montre une forte tension menace / désir, notamment avec les oppositions entre noir et rouge, jeune et vieux, lumière intérieure et nuit extérieur. Le jeune homme habillé en noir et la femme habillée en rouge, sont-ils le remake moderne du petit chaperon rouge? Quoiqu'il en soit, cette mise en scène, impensée au moment de la réalisation puisque les personnes ont tourné avec leurs habits de ville, explique une partie du malaise ressenti lors de la diffusion du film : les participants du focus group ont été perturbés au point de focaliser leur attention sur la situation (et les imaginaires à laquelle elle renvoie) plutôt que le produit/service mis en scène. L'enjeu est donc de maîtriser autant les contenus écrits que les potentialités de connotation culturelle des contenus plastiques et iconiques. Les usagers, leurs vêtements ainsi que leurs comportements vont être soumis à jugement. De même que les formes et couleurs de l'objet ou de l'interface du service innovant.

Notre constat est qu'il y a nécessité d'une maitrise des questions d'écriture - mise en récit et en forme multimédia - dès lors que le chercheur tend vers de nouvelles formes d'expressions de la connaissance. Or, le passage de discours écrits (rapports de recherche, études de marché, analyse de la concurrence, etc.) à des discours multimédias ne va pas de soi. Du fait de la coupure sémiotique, le fonctionnement de la pensée audioscriptuelle diffère de celui de la pensée audiovisuelle. En effet, l'écriture textuelle repose sur l'usage d'une catégorie de signes, que la sémiotique de Peirce désigne sous le terme de symboles. Dans cette catégorie de signes, le rapport entre le signifiant et le signifié est fixé par convention sociale. Par exemple, les lettres T/A/B/L/E permettent de former le mot "table«. Ce mot désigne une multitude d'objets possibles. Sans plus de précision, il forme chez tout interprétant, une représentation mentale générale qui renvoie à quatre pieds et un plateau. Ce qu'en terme Peircien, se nomme un argument. L'utilisation verbale ou par écrit du signifiant "table« n'engage guère de polysémie. Par contre, dès qu'on change de système de signe et opte par exemple pour une représentation visuelle, un problème va se poser. Quel modèle de table utiliser? Si on utilise une table d'écolier, le risque est de focaliser l'attention de l'interlocuteur sur une connotation particulière : celle de l'école, de l'apprentissage. Si on opte pour une table de cuisine, on peut involontairement désigner un contexte culturel particulier qu'on ne désire pas exprimer. L'image se classe alors dans la catégorie des signes iconiques, c'est-à-dire en rapport de ressemblance entre le signifiant et le signifié. Pire encore, dans ces images peuvent se glisser des catégories 
de signe qu'on appelle indices et qui peuvent charger la table de connotation. Par exemple, si sur la table d'écolier je note des tags, des inscriptions, quel surplus de sens peuvent-ils donner à la représentation?

\section{Conclusion}

Les sociologues praticiens, ainsi que la majorité des équipes qui interviennent dans les processus de conception d'innovations technologiques sont amenés à modifier peu à peu leurs pratiques, du fait d'un contexte de travail singulier, tant sur le plan des sujets et des objectifs traités (imaginer des innovations à venir et faire du concret - des objets -) que sur le plan des pratiques professionnelles (où l'interdisciplinarité est rendue presque obligatoire). A cela s'ajoute un environnement en profonde mutation, qui font des outils numériques des compagnons du quotidien, dans la sphère privée autant que professionnelle. La recherche est transformée par l'immersion dans de tels domaines. Mais, à l'inverse, les supports numériques transforment aussi la recherche, dans le sens où ils forcent l'interdisciplinarité : en faisant dialoguer constamment textes images et sons, ils obligent chaque chercheur à entrer dans la compréhension du monde des images, des représentations qui viennent enrichir leurs textes. En conséquence de quoi le chercheur est, de fait, amené à avoir un regard réflexif sur sa pratique, avec l'idée que le dialogue passant par ces objets numériques, nourrit en retour sa discipline de référence. Ainsi, dans ce monde de la "convergence disciplinaire", et dès lors que les équipes tendent vers de nouvelles formes d'expressions de la connaissance, il est nécessaire de maîtriser aussi la mise en récit et en forme multimédia des usages : l'importance de la compétence sémiotique s'avère ici primordiale pour mettre en discussion les univers de la production et ceux de la réception, qui ont en commun des imaginaires sociaux liés aux objets, aux êtres, aux actions, aux affects et à la société elle-même. En un sens, seule l'interdisciplinarité autour d'une même thématique, y compris pour sa médiatisation, devient un "garde-fou méthodologique", à condition de mobiliser et de faire dialoguer les bonnes compétences, au moment adéquat. Elle permet d'éviter les erreurs de sens et d'interprétation.

\section{BIBLIOGRAPHIE}

Anjembe, E. en cours. La projection d'usage des TICs, Retours vers le futur d'une pratique de la sociologie, thèse de doctorat de science politique, Laboratoire PACTE/CNRS, Grenoble Université.

Barthes, R. 1968. «L'Effet de réel », Communications n 11:84-9.

Bowker, G. et Star, S.L. 1999. Sorting Things Out: Classification and Its Consequences, Boston, MIT

Press.

Habermas, J. 1981. Théorie de l'agir communicationnel, titre original : Theorie des kommunikativen Handelns, $2 \mathrm{t}$. 
Habermas, J. 1978. L'Espace Public. Archéologie de la Publicité comme dimension constitutive de la société bourgeoise (1962), trad. française de Marc B. de Launay, éditions Payot, Paris.

Joüet, J. 2000. Retour critique sur la sociologie des usages. Réseaux, 18(100), 487-521. Retrouvé de http://www.persee.fr/web/revues/home/prescript/article/reso_0751-7971_2000_num_18_ 100_2235

Le Marec, J. 2001. «L'usage et ses modèles : quelques réflexions méthodologiques », Spirales, no $28: 105-122$.

Lee, C. P. 2007. «Boundary Negotiating Artifacts: Unbinding the Routine of Boundary Objects and Embracing Chaos in Collaborative Work ", Comput. Supported Coop. Work, vol. 16, noํ 3 : 307-339.

Patenaude, J. and Boissy, P. 2009. L'accompagnement du développement des nanotechnologies par les sciences humaines : un enjeu de recherche », Les nanotechnologies, développement, enjeux sociaux et défis éthiques, Presses de l'Université Laval, sous la direction de JP Béland et J Patenaude : 81-100.

Piriou, O. 2008. « Le nouveau tournant de la sociologie en France dans les années 2000 », Sociologies Pratiques, ㄲo $16: 123-130$.

Trompette, P. et Blanco, E. 2009. L'usage en conception. Projeter sans fermer la carrière sociale des innovations. Dans In Chalas Y. Gilbert C. Vinck D. (Dir.) Comment les acteurs s'arrangent avec l'incertitude, Paris, Editions des Archives Contemporaines : 97-116. Retrouvé http:// books.google.fr/books?id=odpIblVr1VAC\&lpg=PA97\&ots=bNCXSte_Cx\&lr\&pg=PA 97\# $\mathrm{v}=$ onepage \&q\&f=false

Verchère, C. et Anjembe, E. 2010. De la difficulté de fabriquer des objets-frontières. Revue d'anthropologie des connaissances, 4(1) : 36-64.

Vinck, D. 2007. Sciences et société. Sociologie du travail scientifique, Paris, A. Colin.

Von Hippel, E. 2005. Democratizing innovation. The MIT Press.

\section{NOTES}

1. http://www.umanlab.com/. Uman Lab [Usage, Marché, Attitudes \& Nouvelles technologies] est une équipe de recherche technologique (ERT) associé au laboratoire PACTE (2009-2011).

2. http://www.minatec.com/. MINATEC constitue un campus d'innovation unique en Europe et au meilleur rang international dans le domaine des micro et nanotechnologies : 2400 chercheurs, 1200 étudiants et 600 industriels et spécialistes du transfert technologique regroupés sur 20 hectares et disposant de lourdes infrastructures, notamment $10000 \mathrm{~m}^{2}$ de salles blanches.

3. La construction des scénarios d'usage se fonde sur l'école sémiotique de Paris (publications de Algirdas Greimas et Joseph Courtés dans les années 1960-70). La structuration de différents niveaux dans les scénarios a été explicitée par EVERAERT DESMET, N. 1988. Sémiotique du récit, De Boeck-Wesmae. Voir aussi FIELD, S. 1988. Scénario. Les bases de l'écriture scénaristique, Editions Dixit : 272 p. Les scénarios d'usage peuvent être classés dans la catégorie des scénarios dits "d'interaction" : il s'agit de mettre en scène des situations vraisemblables et plausibles dans lesquelles agissent des personnes avec un dispositif innovant. Le but est d'anticiper des usages futurs ou encore d'évaluer la pertinence de ces usages " projetés ». Les scénarios sont destinés aux concepteurs ou tout acteur du domaine de l'innovation. L'avenir est envisagé sous une forme vraisemblable à partir des pratiques existantes. Pour plus de précision: voir le rapport de recherche UMAN LAB, Equipe de Recherche Technologique, Navarro, O et Forest, F et Lavoisy, O C 
et Chanal, V. Sept.2009. "L'utilisation du scénario dans le processus d'innovation: une lecture interdisciplinaire", Grenoble Institut de l'Innovation, Université Pierre Mendès France.

\section{RÉSUMÉS}

Depuis quelques années, les grandes entreprises, notamment celles qui sont engagées dans le numérique, ont renouvelé leur manière de "faire de l'innovation" en prenant en considération les usagers, mais aussi en faisant appel à des compétences nouvelles (sociologie, design, ergonomie). Ce “tournant praticien" n'est pas sans effet sur les pratiques des chercheurs "embarqués" dans ces processus. Notre équipe de recherche s'est penchée sur la question de la représentation et de la circulation de la connaissance dans les projets de conception d'innovations. Il s'agit d'interroger en quoi les différents supports multimedia utilisés peuvent faciliter la formation, la collecte et la transmission de connaissances entre pairs, l'interdisciplinarité, voire la prise en compte de la parole “d'usagers". Les premiers retours montrent que cette démarche amène les chercheurs à un double travail de création: sur le contenu, et sur la manipulation des supports numériques. Ils font aussi état des difficultés de maîtrise de tels supports. Ainsi, la compétence sémiotique s'avère primordiale afin de penser l'écriture propre à ces supports : la « convergence disciplinaire » devient de fait une nécessité.

In recent years, major companies, especially those engaged in digital technologies, have renewed their way to "make innovation« taking into consideration the users, but also using new skills (sociology, design, ergonomics). This "change in practices" is not without effect on the practices of researchers "embedded" in these processes. Our research team focused on the issue of the representation and circulation of knowledge in project design innovations. It examined how the various multimedia tools can facilitate interdisciplinarity, the creation, collection and transmission of knowledge among peers, and even the consideration of the "users«. The first feedback shows that this approach leads researchers to double creation: the content and the manipulation of digital media. But there are also the difficulties of controlling such media. Thus, the semiotic competence appears crucially important to assess the specificity of writing with these tools: "disciplinary convergence« is thus becoming a necessity.

Desde algunos años, las grandes empresas, y especialmente aquéllas vinculadas con lo digital, han renovado su forma de "innovar" tomando en consideración los usuarios y empleando nuevas competencias (sociología, diseño, ergonomía). Este "giro práctico" tiene efectos en las prácticas de los investigadores involucrados en dichos procesos. Nuestro equipo de investigación se ha centrado en la cuestión de la representación y de la circulación del conocimiento en los proyectos de concepción de innovaciones. Nos hemos preguntado en qué medida los diferentes soportes multimedia utilizados pueden facilitar la formación, la colecta y la transmisión de conocimientos entre colegas, la interdisciplinariedad, e incluso la toma en consideración de la opinión de los "usuarios". Los primeros datos de esta investigación ponen en evidencia que esta perspectiva lleva los investigadores a realizar un doble trabajo de creación: sobre el contenido y sobre la manipulación de los soportes digitales. También ponen de relieve las dificultades en el control de dichos soportes. Así, la competencia semiótica aparece como un aspecto primordial para pensar la escritura propia de estos soprotes: la "convergencia disciplinar" se convierte en una necesidad. 
INDEX

Mots-clés : usage, imaginaire, approche communicationnelle, interdisciplinarité, innovation, multimédia, scénario d'usage

Palabras claves : uso, imaginario, perspectiva comunicacional, interdisciplinariedad, innovación, multimedia, guión de uso

Keywords : use, imaginary, communicational approach, interdisciplinarity, innovation, multimedia, scenario based design

\section{AUTEURS}

\section{CÉLINE VERCHÈRE}

Commissariat à l'énergie atomique et aux énergies alternatives (CEA), MINATEC Campus, chercheure associée PACTE/CNRS

celine.verchere@cea.fr

\section{LAURENT COLLET}

Maître de conférences en sciences de l'information et de la communication, directeur de l'UFR Ingémédia à l'Université du Sud Toulon Var, chercheur I3M

laurent.collet@univ-tln.fr 